OPEN ACCESS

Edited by:

Ryan Ceddia,

Vanderbilt University Medical Center,

United States

Reviewed by:

Kamal Rahmouni,

The University of lowa, United States

Michael Zemel,

NuSirt Biopharma, Inc, United States

${ }^{*}$ Correspondence:

Chun Chen

chenchun69@126.com

Chenju Y

yichj@mail.sysu.edu.cn

tThese authors have contributed equally to this work

¥These authors share senior authorship

Specialty section:

This article was submitted to Metabolic Physiology, a section of the journa Frontiers in Physiology

Received: 25 April 2021 Accepted: 04 June 2021 Published: 01 July 2021

Citation:

Zeng $Y$, Huang $T$, Wang $N, X u Y$, Sun $C$, Huang $M$, Chen $C$, Oliver $B G$,

Yi C and Chen H (2021) L-Leucine Improves Metabolic Disorders in Mice

With in-utero Cigarette Smoke Exposure. Front. Physiol. 12:700246. doi: 10.3389/fphys.2021.700246

\section{L-Leucine Improves Metabolic Disorders in Mice With in-utero Cigarette Smoke Exposure}

\author{
Yunxin Zeng ${ }^{1 \dagger}$, Taida Huang ${ }^{1 \dagger}$, Nan Wang ${ }^{1}, Y_{i} X^{1}{ }^{1}$, Chunhui Sun ${ }^{1}$, Min Huang ${ }^{1}$, \\ Chun Chen ${ }^{1 *}$, Brian G. Oliver ${ }^{2,3}$, Chenju Yi ${ }^{1 * \neq}$ and Hui Chen ${ }^{2 \neq}$ \\ ${ }^{1}$ Research Center, The Seventh Affiliated Hospital of Sun Yat-sen University, Shenzhen, China, ${ }^{2}$ Faculty of Science, School of \\ Life Sciences, University of Technology Sydney, NSW, Australia, ${ }^{3}$ Respiratory Cellular and Molecular Biology, Woolcock \\ Institute of Medical Research, Sydney, NSW, Australia
}

Objectives: Maternal cigarette smoke exposure (SE) causes intrauterine undernutrition, resulting in increased risk for metabolic disorders and type 2 diabetes in the offspring without sex differences. L-leucine supplementation has been shown to reduce body weight and improve glucose metabolism in both obese animals and humans. In this study, we aimed to determine whether postnatal L-leucine supplementation in female offspring can ameliorate the detrimental impact of maternal SE.

Methods: Female Balb/c mice (6-week) were exposed to cigarette smoke (SE, 2 cigarettes/day) prior to mating for 5 weeks until the pups weaned. Sham dams were exposed to air during the same period. Half of the female offspring from the SE and SHAM dams were supplied with L-leucine via drinking water $(1.5 \% \mathrm{w} / \mathrm{w})$ after weaning (21-day) for 10 weeks and sacrificed at 13 weeks (adulthood).

Results: Maternal SE during pregnancy resulted in smaller body weight and glucose intolerance in the offspring. L-leucine supplement in Sham offspring reduced body weight, fat mass, and fasting blood glucose levels compared with their untreated littermates; however somatic growth was not changed. L-leucine supplement in SE offspring improved glucose tolerance and reduced fat mass compared with untreated littermates.

Conclusions: Postnatal L-leucine supplement could reduce fat accumulation and ameliorate glucose metabolic disorder caused by maternal SE. The application of leucine may provide a potential strategy for reducing metabolic disorders in offspring from mothers who continued to smoke during pregnancy.

Keywords: maternal smoking, glucose intolerance, insulin, fat mass, leucine

\section{INTRODUCTION}

Tobacco smoking is a global public health risk. According to the WHO, in 2016, the smoking rate in females over 15 years old was 20.7\% in Europe, $12.4 \%$ in America, and $1.9 \%$ in China, while the second-hand smoking rate in females was as high as $46.9 \%$ in China (Ding et al., 2016; World Health Organization, 2019). Maternal cigarette smoke exposure (SE), including direct and second-hand smoking during pregnancy, is a major cause of intrauterine undernutrition resulting in 
several adverse health outcomes, including preterm birth, low birth weight, and catch-up growth in childhood (Collaco et al., 2017; Wagijo et al., 2017). In addition to these short-term adverse effects, offspring from mothers exposed to cigarette smoke during pregnancy are also more likely to develop metabolic disorders in adulthood, such as glucose intolerance, type 2 diabetes, fatty liver changes, dyslipidemia, and cardiovascular disease (Mendez et al., 2008; Gorog et al., 2011; La Merrill et al., 2015).

Around 463 million people suffering from diabetes, which is one of the greatest public health challenges in both developed and developing countries (Saeedi et al., 2019). The incapability of pancreatic $\beta$-cells to produce adequate insulin or reduced insulin response in glucose deposit tissues leads to a chronic increase in blood glucose level followed by vascular damages which are the prime cause of death in diabetic patients (Bennett et al., 2018; Zheng et al., 2018). Previous studies in rodent models have shown that maternal nicotine treatment interrupts $\beta$-cell functions in offspring, and maternal SE induced intrauterine undernutrition is also associated with overconsumption in offspring, increasing the risk of insulin resistance and glucose intolerance (Holloway et al., 2005; Bruin et al., 2008, 2010; Sullivan et al., 2014). Therefore, to reduce the risk of metabolic disorder and diabetes, it is crucial to find effective preventive strategies to ameliorate the adverse impacts of maternal SE on offspring.

In the study of diabetes prevention, a high-protein diet has been proven to produce better glycemic control, fat loss, and preservation of muscle mass than a carbohydraterich diet with the same caloric intake (Skov et al., 1999; Parker et al., 2002; Layman et al., 2003). During this process, branch chain amino acids, especially L-leucine, have been suggested to play a critical role. Leucine is an essential amino acid and can only be obtained from the diet. In the brain, L-leucine activates the mammalian target of rapamycin in the hypothalamus (Cota et al., 2006), resulting in its beneficial effects of inducing weight loss, and improving glucose homeostasis in mouse models of obesity and diabetes (Arakawa et al., 2011; Chen et al., 2012; Westerterp-Plantenga et al., 2014). This evidence suggests that leucine supplement can be a potential strategy to prevent glucose disorders due to maternal SE.

The regulatory network for energy homeostasis has been widely studied in the hypothalamus where the neurons produce the appetite stimulator neuropeptide Y (NPY), and appetite suppressor $\alpha$-melanocyte stimulating hormone cleaved from proopiomelanocortin (POMC) (Morton et al., 2006). Chemicals in cigarette smoke can inhibit hypothalamic NPY level and the same can happen in fetal brain and suckling pups when they continuously receive the chemicals from breastmilk. When the suckling pups are weaned, it can induce quitting smoking type of rebound response of NPY to cause overeating in childhood, similar to what happens to smokers when they quit smoking (Grove et al., 2001). In contrast, an intracerebroventricular administration of L-leucine, could reduce food intake and body weight in chow-fed animals, due to its suppression of hypothalamic NPY expression (Cota et al., 2006). Dietary leucine can pass the blood-brain barrier to access the brain (Chen et al.,
2012); however, in the situation of high fat diet consumption, oral leucine supplement was not able to affect the adiposity but still can improve glycemic control (Chen et al., 2012).

While it is increasingly recognized the importance of the fetal environment on the susceptibility to future metabolic disorders, it is also important to look for effective early interventions to prevent adverse maternal impact in the offspring. Therefore, we hypothesized that in offspring from dams exposed to cigarette smoke during pregnancy, postnatal L-leucine supplement in drinking water will prevent excess fat accumulation and benefit glycemic control in adulthood. To address this hypothesis, we used our published mouse model of maternal SE (Huang et al., 2021) and supplied Lleucine in the drinking water to the female offspring from weaning for 10 weeks until they reached adulthood. As there is no sexual difference in metabolic disorders due to maternal SE, we only studied females. We aimed to investigate the effects of maternal SE and postnatal leucine supplement on body weight, adiposity, glucose tolerance, and the gene expression of metabolic markers in the hypothalamus and fat tissues.

\section{MATERIALS AND METHODS}

\section{Animal Experiments}

According to the previous publication on the strain dependence of the response to cigarette smoke exposure (Vlahos et al., 2006), female Balb/C mice (6-week-age) were used. They were housed at $20 \pm 2{ }^{\circ} \mathrm{C}$ and maintained on a $12: 12 \mathrm{~h} \mathrm{light/dark} \mathrm{cycle} \mathrm{(lights}$ on 6:00 a.m.) with ad libitum access to standard rodent chow and water. All the animal experiments were approved by the Animal Ethics Committee of the San Yet-sun University (SYSUIACUC-2020-81060) and followed the guidelines for animal care and use for scientific research by the National Institute of Health, USA.

After acclimatization, mice with similar body weight were randomly assigned to two groups, sham exposure (SHAM) and SE. The SE group was exposed to smoke produced by 2 cigarettes (Double Happiness; Tar: $8 \mathrm{mg}$; nicotine: $0.7 \mathrm{mg}$; CO: $12 \mathrm{mg}$ ) inside a perspex box (18 liters) in the fume hood, twice a day for 5 weeks before mating and during the gestation and lactation periods following a published protocol as we have previously published (Huang et al., 2021). The breeders in the SHAM group were treated identically except for the exposure to the air. After 5 weeks of preconditioning, females were placed with male mice to mate (ratio 3:1). All breeders continuously received the same treatments until pups were weaned. The pups and male breeders remained in their home cages when the females were treated and not subjected to any exposure.

\section{Post-weaning L-Leucine Supplement in Female Offspring}

One day after birth, litter sizes were adjusted to 4-6 pups (sex ratio 1:1) to minimize the impact of milk competition. The pups were weaned at 21 days of age. Since there is no sexual difference in the metabolic disorders due to maternal SE, only 
female offspring were used in the present study (males were subjected to a different study). Within each litter, half of the pups were supplied with L-leucine (Sigma, St Louis, MO, USA) via drinking water $(1.5 \% \mathrm{w} / \mathrm{w})$ for 10 weeks. Normal drinking water was provided to the other half of the litter. This yielded four experimental groups, SHAM, SHAM-leucine, SE, and SEleucine. Water intake was measured 1 week before the endpoint by recording the difference between the weight of the water bottle at a $24 \mathrm{~h}$ interval.

\section{Intraperitoneal Glucose Tolerance Test (IPGTT)}

IPGTT was performed in the offspring at 12 weeks of age as previously described (Nguyen et al., 2015). In brief, after $5 \mathrm{~h}$ of fasting, the baseline of glucose level was measured in blood samples collected from the tail tip $\left(\mathrm{T}_{0}\right)$ by Accu-Chek ${ }^{\circledR}$ glucose meter (Roche, Germany). After glucose injection ( $2 \mathrm{~g} / \mathrm{kg}$, ip), the same measurement was performed again at $15,30,60$, and $90 \mathrm{~min}$ to calculate the area under the curve (AUC) for each mouse.

\section{Sample Collection}

Female offspring were deeply anesthetized (ketamine/xylazine $180 / 32 \mathrm{mg} / \mathrm{kg}$ ) at 13 weeks after overnight fasting. Blood glucose levels were immediately measured by Accu-Chek ${ }^{\circledR}$ glucose meter after blood collection. Plasma was stored at $-20^{\circ} \mathrm{C}$ for the measurement of insulin levels using a commercially available ELISA kit following the manufacture's instruction (Abnova, Taiwan). The Homeostatic Model Assessment of Insulin Resistance (HOMA-IR) was calculated according to the formula: insulin $(\mu \mathrm{U} / \mathrm{mL}) \times$ glucose $(\mathrm{mM}) / 22.5$. Thereafter, mice were subsequently sacrificed by decapitation. The tissues including the hypothalamus, body fat (brown fat, epididymal, retroperitoneal, and visceral fat), organs (liver, kidney, and heart), as well as skeletal muscle (soleus, extensor digitorum longus (EDL), and quadratus lumborum) were dissected and weighed. Hypothalamus, retroperitoneal fat, and brown fat were stored at $-80^{\circ} \mathrm{C}$ for subsequent measurement of mRNA expression of metabolic markers.

\section{Quantitative Real-Time PCR Assays}

Total RNA was isolated from individual samples by TriZol Reagent following the manufacture's protocol [Invitrogen, United State of America (USA)]. The purified total RNA was used for First-strand cDNA generation with a synthesis kit (One-step gDNA Removal, TransGen Biotech, China). TaqMan probe/primers were pre-optimized and validated by the manufacturer (Thermo Fisher Scientific, USA) were used for quantitative real-time PCR (CFX96, Bio-Rad, USA). Target metabolic marker genes included neuropeptide Y (Npy: Mm00445771_m1), neuropeptide Y1 receptor (Npy1r: Mm00445771_m1), proopiomelanocortin (Pomc: Mm00435874_m1), melanocortin-4 receptor: (Mc4r: Mm00457483_s1), single minded gene 1 (Sim1: Mm00441390_m1), monocarboxylic acid transporters 2 (Mct2: Mm00441442_m1), monocarboxylic acid transporters 4 (Mct4: Mm01246824_g1), lactate dehydrogenase B (Ldhb: Mm05874166_g1) in hypothalamus and carnitine palmitoyltransferase 1 alpha (Cpt1 $\left.\alpha: M m 00550438 \_m 1\right)$, tumor necrosis factor alpha (Tnfo: Mm00443259_g1), adipose triglyceride lipase (Atgl: Mm01275939_g1), as well as uncoupling protein 1 (Ucp1: Mm00494069_m1) in BAT.

\section{Statistical Analysis}

All the data are presented as mean \pm SEM. Differences between the groups were analyzed using two-way ANOVA, except for

TABLE 1 | Effects of maternal se and postnatal leucine supplement in female offspring.

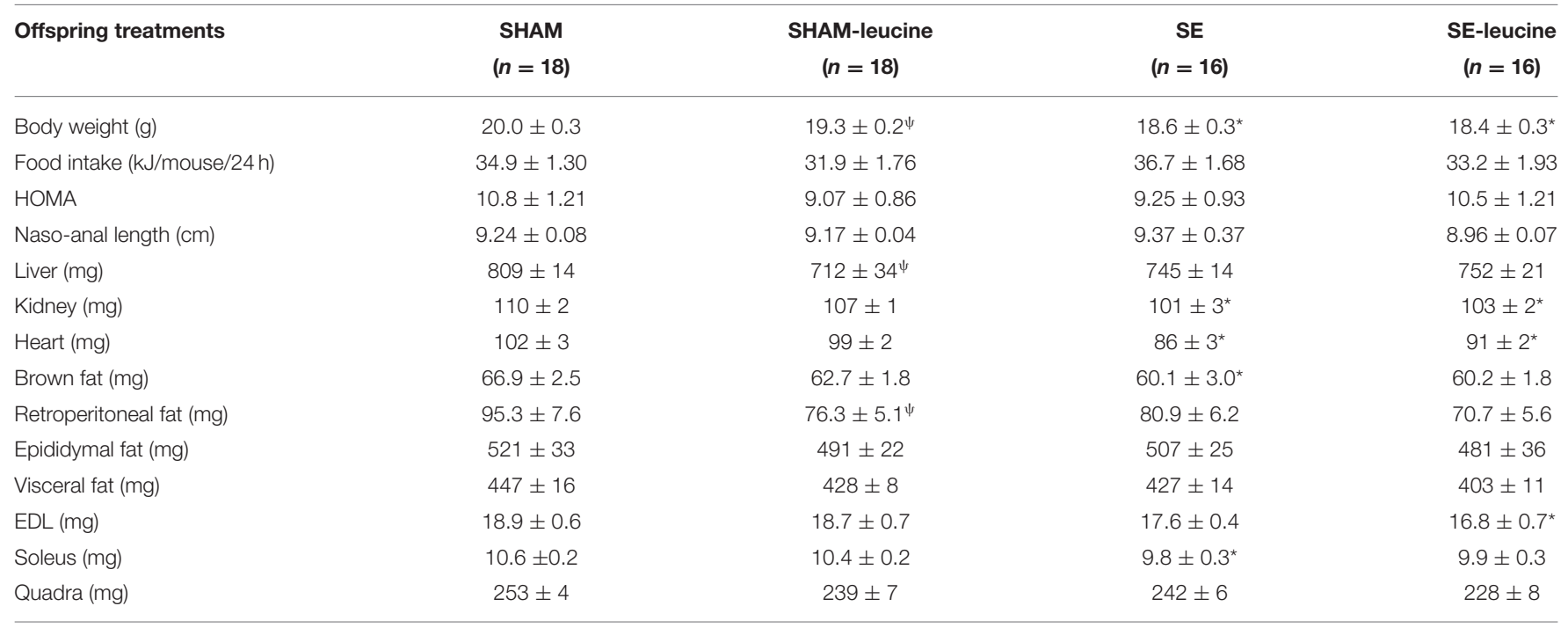

Results are expressed as mean \pm SEM, and analyzed by two-way ANOVA, followed by post hoc LSD tests. ${ }^{*} P<0.05$, maternal $S E$ effect; ${ }^{*} P<0.05$, leucine effect. EDL, extensor digitorum longus; Quadra, quadratus lumborum muscle; SE, smoke exposure. 

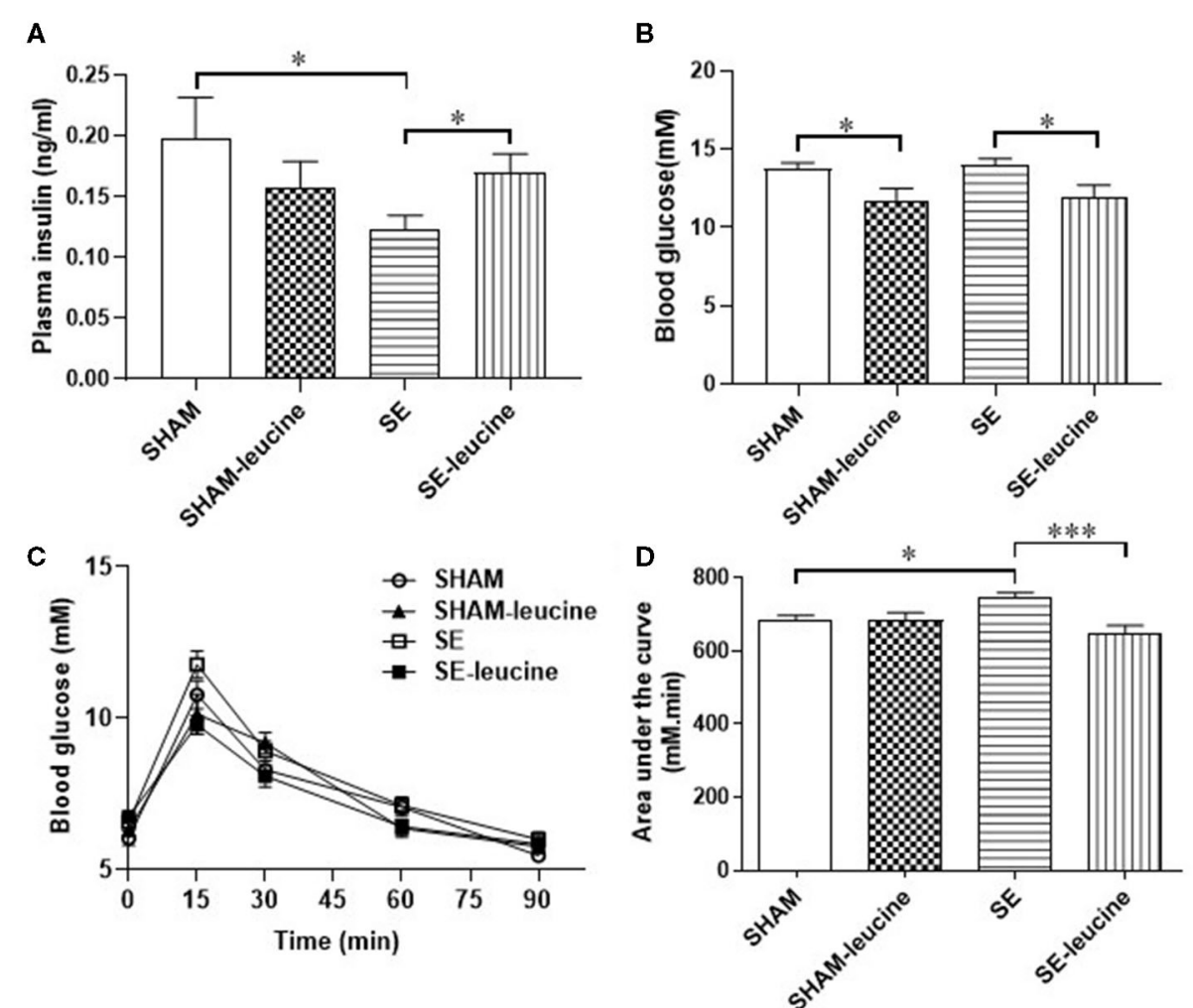

FIGURE 1 | Blood glucose metabolic profile in female offspring. Plasma insulin (A) and blood glucose (B) level in female offspring at 13 weeks. Blood glucose levels during Intraperitoneal glucose tolerance test (IPGT, C) and the area under the curve (D) at 12 weeks. Results are expressed as the mean \pm SEM $(n=8)$, and analyzed by two-way ANOVA, followed by post-hoc LSD tests. ${ }^{*} P<0.05,{ }^{\star \star \star} P<0.001$.

glucose level during IPGTT which were analyzed by multifactor ANOVA, followed by post-hoc LSD tests, if the data were normally distributed. If not, data were log-transformed to achieve normality of distribution before they were analyzed. $P<0.05$ was considered statistically significant.

\section{RESULTS}

\section{Effects of Maternal SE and Postnatal L-Leucine Supplement on Anthropometry}

At 13 weeks, mice in the SE groups showed smaller body weight than those in the SHAM group $(P<0.05$, Table 1$)$, consistent with the literature (Chan et al., 2016). Maternal SE also significantly reduced the weights of vital organs and skeletal muscle soleus $(P<0.05$, SHAM vs. SE, Table 1). Although white fat masses also appeared smaller in the SE offspring, they did not reach statistical significance.

L-leucine supplement only significantly reduced body weight, liver weight, and retroperitoneal white fat mass in the SHAM offspring $(\mathrm{P}<0.05$, SHAM-leucine vs. SHAM, Table 1$)$. Such treatment did not significantly affect any of the anthropometric markers in the SE offspring, albeit smaller white fat mass (Table 1).

Neither maternal SE nor post-weaning L-leucine supplement affected food intake and somatic growth reflected by naso-anal length (Table 1). The water intake was similar between SHAMleucine $(3.62 \pm 0.02 \mathrm{ml} /$ mouse/day) and SE-leucine $(3.17 \pm$ $0.47 \mathrm{ml} /$ mouse/day) groups in adulthood. This equivalent to a leucine intake of $54.3 \mathrm{mg} / \mathrm{mouse} /$ day and $47.6 \mathrm{mg} / \mathrm{mouse} /$ day at this time point.

\section{Leucine Supplement Normalized the Glucose Intolerance Caused by Maternal SE}

At 13 weeks, plasma insulin level was significantly decreased in the SE group than that in the SHAM group $(P<0.05$, SHAM vs. SE, Figure 1A), although fasting blood glucose levels were similar between the groups (Figure 1B). In addition, adult SE offspring exhibited impaired ability to clear blood glucose during IPGTT compared with the SHAM offspring, reflected by the higher AUC values $(P<0.05$, SHAM vs. SE, Figures 1 C,D).

The supplement of leucine normalized plasma insulin level in the SE offspring $(P<0.05$, SE-leucine vs), reduced fasting blood glucose in both SHAM and SE offspring $(P<0.05$ vs. untreated littermates, Figure 1B). Moreover, leucine supplement effectively normalized glucose tolerance in the SE offspring ( $P$ $<0.001$, SE vs. SE-leucine, Figure 1D). However, systematic glucose metabolism seems not to be related to insulin resistance, as HOMA index was not significantly changed by maternal SE or leucine supplement (Table 1). 


\section{L-Leucine Supplement Restored Pomc Expression in the Hypothalamus of SE Offspring}

To evaluate the effects of the maternal SE and post-weaning leucine supplement on the feeding regulators, we measured food intake and analyzed the mRNA expression of classical genes involved in appetite regulation in the hypothalamus. There was no difference in food intake among the groups (Table 1). In the SE offspring, the levels of hypothalamic Npy and Npy1r mRNA expression were similar to those in the SHAM offspring (Figures 2A,B), whereas the Pomc expression was markedly downregulated $(P<0.05$, SHAM vs. SE, Figure $2 \mathrm{C})$. However, the expression of $M c 4 r$, which is the dominant anorexigenic receptor for Pomc derived $\alpha$-melanocyte-stimulating hormone, its downstream signaling Sim1, as well as lactate transports Mct2 and Mc4 and Ldhd (involved in lipid metabolism) was not affected by maternal SE (Figures 2D-H), in line with unchanged food intake.

Postweaning supplement of L-leucine only normalized Pomc expression in SE-leucine group $(p<0.01$, SE vs. SE-leucine, Figure 2C). It did not significantly change any of the other metabolic regulators in the hypothalamus measured in this study, although there is a trend in increased Mct4 in both SHAM and SE offspring ( $~ 50 \%$ increase compared with their untreated littermates, Figure 2G). However, food intake was not significantly changed by leucine supplement (Table 1).

\section{Effects on the Substrate Metabolic in the Fat in Female Offspring}

In the SE group, the expression of the thermogenesis markers Ucp 1 was downregulated by maternal SE $(P<0.05$, Figure 3A). There was a trend decrease in the expression of Atgl in retroperitoneal fat by maternal, but the difference was not statistically significant (Figure 3B). The expression of $C p t 1 \alpha$, a rate-limiting regulator for fatty acid $\beta$-oxidation in mitochondria, and inflammatory marker Tnf $\alpha$ was not affected by maternal SE (Figures 3C,D).

L-leucine supplement reduced Ucp1 expression only in the SHAM offspring $(P<0.05$, SHAM-leucine vs. SHAM), and increased Atgl expression only in the SE offspring $(P<0.01$, Figure 3B). Interestingly, although leucine showed no impact on Cpt1 $\alpha$ expression in either SHAM or SE offspring, its level was significantly lower in SE-leucine mice compared to SHAM-leucine group $(P<0.05$, SHAM-leucine vs. SE-leucine, Figure 3A). The expression of Tnfo was not affected by L-leucine supplement (Figure 3D).

\section{DISCUSSION}

The smoking cessation rate among pregnant women is quite low (Coleman et al., 2015). This possesses a significant health risk in their offspring later in life. Finding a non-invasive and effective prophylactic strategy to protect such offspring can significantly improve their quality of life in the long term. The novel approach of this study is the introduction of postweaning leucine supplement in the offspring to ameliorate the adverse impact of maternal SE. We found that leucine supplement normalized glucose metabolic disorders and insulin insufficiency caused by maternal SE in adult offspring. Even in the SHAM offspring, leucine supplement reduced their fat mass and lowered fasting blood glucose without affecting somatic growth, suggesting its benefit to metabolic profile in general.

In this study, we demonstrated the detrimental effect of maternal SE during pregnancy, which led to smaller body weight, glucose intolerance, and lower plasma insulin level in adult offspring. This is consistent with the literature (Saad et al., 2018; Li et al., 2019). Our study also showed long-lasting effects of maternal SE during pregnancy on reduced lean body mass, including the vital organs (e.g., liver) and skeletal muscle soleus. Tobacco smoke contains over 5000 harmful chemicals (Talhout et al., 2011). The nicotine in cigarette smoke was found to reduce the uteroplacental blood flow leading to inefficient nutrition supplement for the growing fetus. Nicotine can also cross the blood-placental barrier to enter the fetal circulation to concentrate higher nicotine level than that in smoking mother, which further disrupted normal development of the endocrine, neurologic, respiratory, and cardiovascular systems (Lisboa et al., 2012; Holbrook, 2016; Jamshed et al., 2020). In addition, CO inhibits oxygen delivery to the cells and cause in utero hypoxia due to a great affinity to hemoglobin and increases carboxyhemoglobin levels in umbilical arteries (Ko et al., 2014). In the present study, fetal underdevelopment was not corrected by so called "catch-up growth" after birth in humans (Al Mamun et al., 2006; Darendeliler, 2019). This may be due to the diet in the animal study, which is standard rodent chow with balanced nutrition; whereas in humans, smoking mothers are more likely to consume foods high in sugar and saturated fat which they also feed to their children (Al Mamun et al., 2006). This dietary choice would encourage the development of obesity in such offspring. Future studies can introduce the second insult of high fat diet consumption in the offspring to further study the mechanism underlying metabolic disorders in this population.

Furthermore, we also observed that maternal SE would induce glucose intolerance together with reduced plasma insulin level, which is consistent with the previous study in both human and mouse models (Henkin et al., 1999; Chen et al., 2011). In rodent models of nicotine exposure during lactation, pancreatic $\beta$-cells depletion with subsequent impaired glucose homeostasis was observed, suggesting the toxic effect of nicotine on $\beta$-cell function (Bruin et al., 2010; Primo et al., 2013). In addition, in the major glucose deposition organ fat tissue, the pro-inflammatory cytokine TNF $\alpha$ can directly block the insulin receptor signaling pathway to cause insulin resistance and glucose intolerance (Montgomery and Ekbom, 2002; Vuguin et al., 2004). However, in this study, the expression of fat $\operatorname{Tnf} \alpha$ was not changed by maternal SE, in line with HOMA index ruling out the involvement of insulin resistance, suggesting insufficient insulin production may be the major driver of glucose metabolism disorder in the offspring with in-utero SE exposure. In humans, maternal smoking is linked to an increased risk of type 2 diabetes independent of diet choices (Jaddoe et al., 2014).

In the SE offspring, the fat mass of all pads collected in this study was reduced although without statistical significance. This 

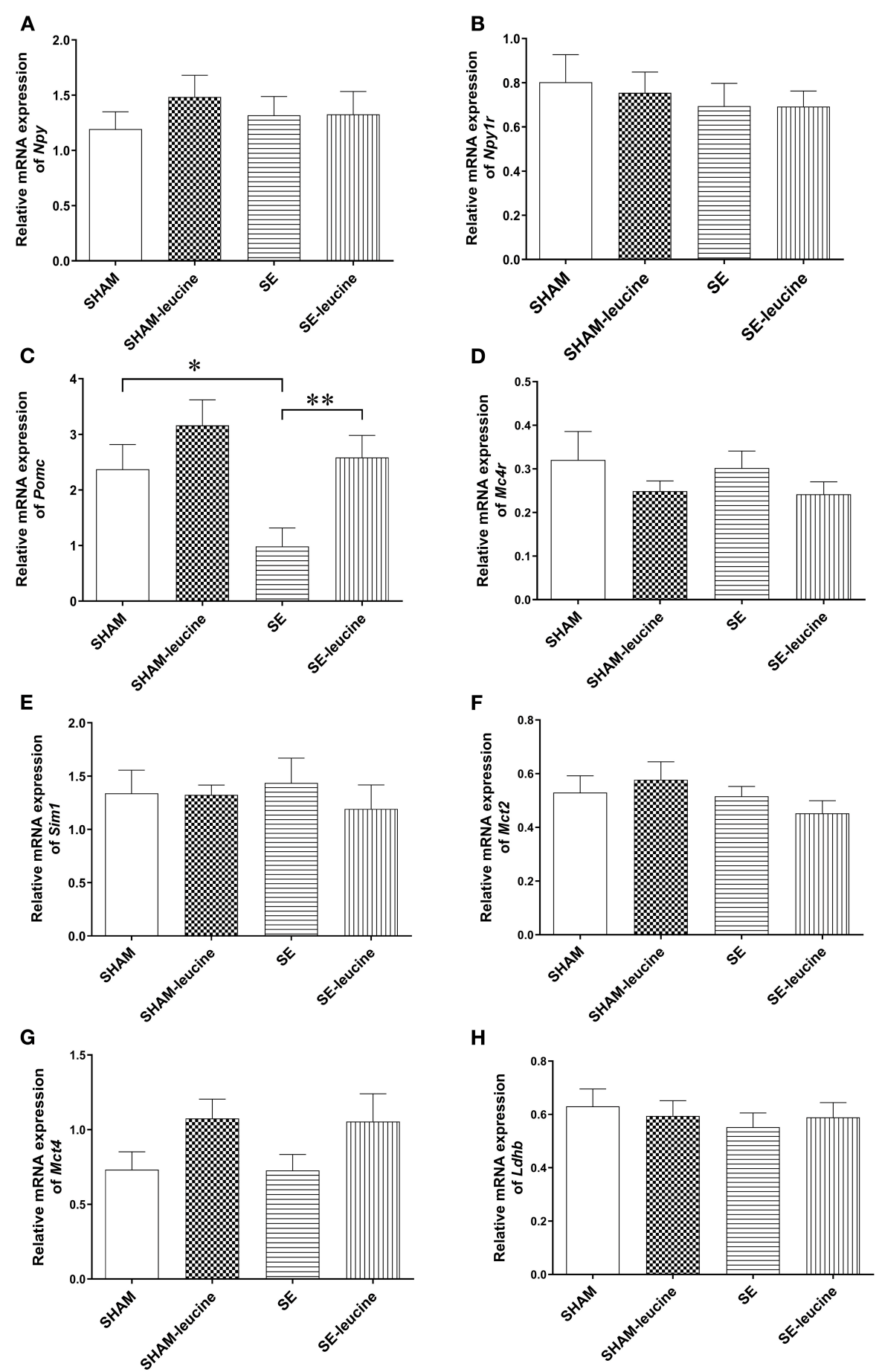

FIGURE 2 | Expression of metabolic regulators in the hypothalamus. The mRNA expression of Npy (A), Npy1r (B), Pomc (C), Mc4R (D), Sim1 (E), Mct2 (F), Mct4 (G), and $L d h b(\mathbf{H})$ in the hypothalamus of female offspring at 13 weeks $(n=8)$. Results are expressed as mean \pm SEM and analyzed by two-way ANOVA, followed by post hoc LSD tests. ${ }^{\star} P<0.05,{ }^{* *} P<0.01$.

may lead to the adaptive reduction in Ucp1 expression. UCP1 mediate non-shivering thermogenesis and basal metabolic rate in brown fat (Cannon and Nedergaard, 2004). Fasting or chronic food restriction can downregulate Ucp1 expression (Cannon and
Nedergaard, 2004; Oelkrug et al., 2015; Peixoto et al., 2020). It is unclear the contribution of downregulated hypothalamic Pomc expression as none of its downstream signaling elements in the regulation of metabolic homeostasis was changed accordingly, 

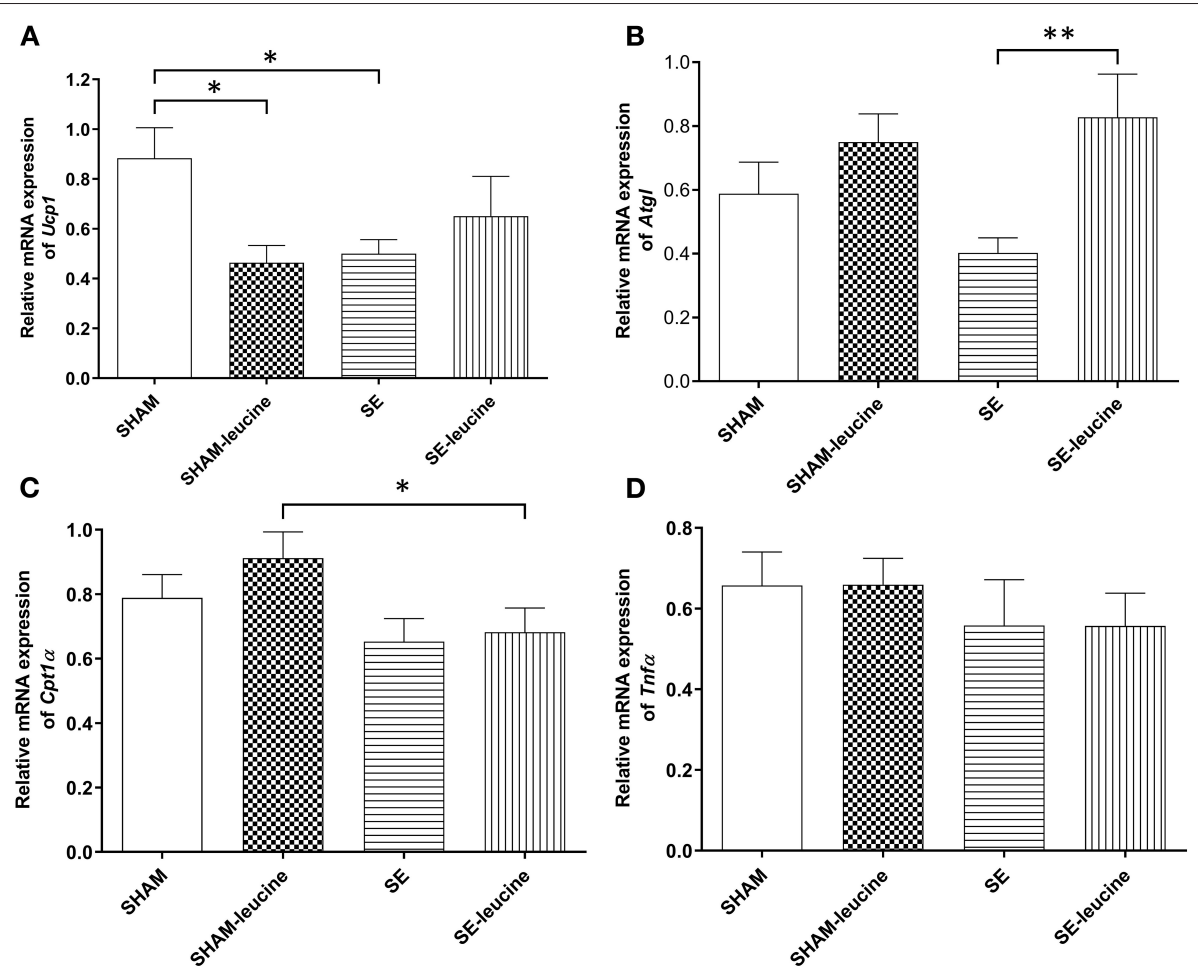

FIGURE 3 | Expression of lipid metabolic markers in the retroperitoneal white fat tissue. mRNA expression of uncoupling protein (Ucp)1 in the brown fat (A), Atgl (B), Cpt1 $\alpha(\mathbf{C})$, and $\operatorname{Tnf} \alpha(\mathbf{D})$ in the retroperitoneal fat, in female offspring at 13 weeks $(n=8)$. Results are expressed as mean \pm SEM. Data were analyzed by two-way ANOVA, followed by post-hoc LSD tests. ${ }^{\star} P<0.05$, ${ }^{\star \star} P<0.01$.

which requires investigations into other functions of Pomc. It may also be an adaptive response to reduced fat mass.

L-leucine supplements have been shown to improve glucose metabolism and insulin sensitivity even in obese rats (Bassil et al., 2007; Berry-Kravis et al., 2007; Chen et al., 2012). Indeed, the most prominent benefit of leucine supplement is perhaps the normalized glucose metabolism in the SE offspring. Since HOMA index was not significantly affected, the mechanism of improved glucose metabolism may not be related to insulin sensitivity, but driven by significantly increased blood insulin levels. This observation is supported by previous studies showing the beneficial effect of leucine on $\beta$-cell function and metabolism (Yang et al., 2010). L-leucine is an essential amino acid that is normally obtained via diet. Considering the independent risk of type 2 diabetes by maternal smoking, human trials can be considered to implement such prophylactic treatment.

Furthermore, in the SHAM offspring, L-leucine administration reduced their body weight and fat mass. As a result, Ucp1 is downregulated to prevent excessive energy expenditure. Previous studies in mice demonstrated that leucine promotes fat loss by increasing fatty acid utilization in adipocytes (Gual et al., 2005; Sun and Zemel, 2007; Zhang et al., 2007). Atgl is an essential enzyme to break down triglycerides into free fatty acids and glycerol and CPT $1 \alpha$ is the rate limiting step for $\beta$-oxidation of fatty acids into ATP in the mitochondria. With L-leucine supplement, both markers were non-significantly increased in the SHAM offspring. It raised the question of whether a statistical significance is required to exert physiological function. Nevertheless, the administration of leucine upregulated Atgl expression, in the face of some reduction in fat mass. In the SE offspring, fat loss was not as prominent as the SHAM offspring. This may be due to the need for a minimum amount of fat tissue to maintain normal metabolic function. Leucine did not show a measurable impact on the key metabolic regulators in the hypothalamus, which is somewhat consistent with our previous study using long-term leucine supplement (Chen et al., 2012). This suggests that peripheral tissues may be the primary targets in such setting. Future studies can also investigate whether this alteration can protect the SE offspring from high fat diet consumption induced obesity.

We need to acknowledge the limitations of this study. We only trialed one dose of L-leucine according to our previous study (Chen et al., 2012) in the female offspring to prove the concept. Previously, leucine supplement was shown to improve glucose metabolic disorders due to maternal obesity in male offspring (Chen et al., 2012; Saad et al., 2018). As this study was only performed on female mice, the positive outcome on glucose metabolism may not happen in the male offspring, who also showed similar metabolic disorders in our previous studies on the same model (Nguyen et al., 2015; Saad et al., 2018; Li et al., 2019; Huang et al., 2021). Future studies can investigate a dose-response curve of such treatment in both male and 
female offspring. In this study, we only measured the classical metabolic regulators, which were not affected by a combination of a nutritional balanced diet and leucine. This may be due to glucose intolerance as the only measurable abnormality in this model. Future studies can induce more severe metabolic disorders by introducing additional risk factors.

\section{CONCLUSION}

L-leucine supplement can reduce fat mass potentially by increasing lipid breakdown and/or metabolism in the fat tissue in both SHAM and SE offspring. Importantly, our study also suggests the benefits of postnatal L-leucine supplement to improve glucose tolerance in SE offspring by improving their blood insulin levels, which may potentially reduce the risk of type 2 diabetes. Future studies can employ additional high fat diet feeding in the SE offspring to confirm the prophylactic effects of L-leucine supplement on the development of obesity and type 2 diabetes.

\section{DATA AVAILABILITY STATEMENT}

The raw data supporting the conclusions of this article will be made available by the authors, with valid justification.

\section{REFERENCES}

Al Mamun, A., Lawlor, D. A., Alati, R., O'callaghan, M. J., Williams, G. M., and Najman, J. M. (2006). Does maternal smoking during pregnancy have a direct effect on future offspring obesity? Evidence from a prospective birth cohort study. Am. J. Epidemiol. 164, 317-325. doi: 10.1093/aje/kwj209

Arakawa, M., Masaki, T., Nishimura, J., Seike, M., and Yoshimatsu, H. (2011). The effects of branched-chain amino acid granules on the accumulation of tissue triglycerides and uncoupling proteins in diet-induced obese mice. Endo. J. 58, 161-170. doi: 10.1507/endocrj.K10E-221

Bassil, M. S., Hwalla, N., and Obeid, O. A. (2007). Meal pattern of male rats maintained on histidine-, leucine-, or tyrosine-supplemented diet. Obesity 15, 616-623. doi: 10.1038/oby.2007.565

Bennett, J. E., Stevens, G. A., Mathers, C. D., Bonita, R., Rehm, J., Kruk, M. E., et al. (2018). NCD Countdown 2030: worldwide trends in non-communicable disease mortality and progress towards sustainable development goal target 3.4. Lancet 392, 1072-1088. doi: 10.1016/S0140-6736(18)31992-5

Berry-Kravis, E., Abrams, L., Coffey, S. M., Hall, D. A., Greco, C., Gane, L. W., et al. (2007). Fragile $\mathrm{X}$-associated tremor/ataxia syndrome: clinical features, genetics, and testing guidelines. Mov. Disord. 22, 2018-2030. doi: 10.1002/mds.21493

Bruin, J. E., Gerstein, H. C., and Holloway, A. C. (2010). Long-term consequences of fetal and neonatal nicotine exposure: a critical review. Toxicol. Sci. 116, 364-374. doi: 10.1093/toxsci/kfq103

Bruin, J. E., Petre, M. A., Lehman, M. A., Raha, S., Gerstein, H. C., Morrison, K. M., et al. (2008). Maternal nicotine exposure increases oxidative stress in the offspring. Free. Radical. Bio. Med. 44, 1919-1925. doi: 10.1016/j.freeradbiomed.2008.02.010

Cannon, B., and Nedergaard, J. (2004). Brown adipose tissue: function and physiological significance. Physiol. Rev. 84, 277-359. doi: 10.1152/physrev.00015.2003

Chan, Y. L., Saad, S., Al-Odat, I., Zaky, A. A., Oliver, B., Pollock, C., et al. (2016). Impact of maternal cigarette smoke exposure on brain and kidney health outcomes in female offspring. Clin. Exp. Pharmacol. Physiol. 43, 1168-1176. doi: 10.1111/1440-1681.12659

Chen, H., Iglesias, M. A., Caruso, V., and Morris, M. J. (2011). Maternal cigarette smoke exposure contributes to glucose intolerance and decreased brain insulin

\section{ETHICS STATEMENT}

The animal study was reviewed and approved by Animal Experimentation Ethics Committee of the San Yet-sun University (number: SYSU-IACUC-2020-81060).

\section{AUTHOR CONTRIBUTIONS}

YZ: methodology, investigation, and writing-original draft. TH: investigation and editing. NW, YX, CS, and $\mathrm{MH}$ : investigation. BO: conceptualization, supervision, and review. CY: conceptualization, supervision, writing, and editing. HC: conceptualization, supervision, writing, and review. All authors contributed to the article and approved the submitted version.

\section{FUNDING}

This study was supported by research grants from the National Nature Science Foundation of China (NSFC 81971309), Guangdong Basic and Applied Basic Research Foundation (2019A1515011333), Fundamental Research Funds for the Central Universities (19ykzd04), and the Shenzhen Fundamental Research Program (JCYJ20190809161405495, JCYJ20190809190601671 and RCYX20200714114644167).

action in mice offspring independent of maternal diet. PLoS ONE 6:e27260. doi: 10.1371/journal.pone.0027260

Chen, H., Simar, D., Ting, J. H. Y., Erkelens, J. R. S., and Morris, M. J. (2012). Leucine improves glucose and lipid status in offspring from obese dams, dependent on diet type, but not caloric intake. J. Neuroendocrinol. 24, 1356-1364. doi: 10.1111/j.1365-2826.2012.02339.x

Coleman, T., Chamberlain, C., Davey, M. A., Cooper, S. E., and LeonardiBee, J. (2015). Pharmacological interventions for promoting smoking cessation during pregnancy. Cochrane. Database. Syst. Rev. 12:CD010078. doi: 10.1002/14651858.CD010078.pub2

Collaco, J. M., Wilson, K. M., and Mcgrath-Morrow, S. A. (2017). More evidence linking smoke-free legislation and lower risk of prematurity and low birth weight. Pediatrics 139:e20170795. doi: 10.1542/peds.2017-0795

Cota, D., Proulx, K., Smith, K. A. B., Kozma, S., Thomas, G., Woods, S., et al. (2006). Hypothalamic mTOR signaling regulates food intake. Science 312, 927-930. doi: 10.1126/science.1124147

Darendeliler, F. (2019). IUGR: Genetic influences, metabolic problems, environmental associations/triggers, current and future management. Best. Pract. Res. Clin. Endocrinol. Metab. 33:101260. doi: 10.1016/j.beem.2019.01.001

Ding, R., Wang, P., Ma, L., and Tian, Y. (2016). A meta-analysis on the prevalence of secondhand smoke among Chinese mainland female residents. Prev. Med. 28, 671-676. Available online at: http://www.zjyfyxzz.com/CN/Y2016/V28/I7/ 671

Gorog, K., Pattenden, S., Antova, T., Niciu, E., Rudnai, P., Scholtens, S., et al. (2011). Maternal smoking during pregnancy and childhood obesity: results from the CESAR study. Matern. Child. Health. J. 15, 985-992. doi: 10.1007/s10995-009-0543-5

Grove, K. L., Sekhon, H. S., Brogan, R. S., Keller, J. A., Smith, M. S., and Spindel, E. R. (2001). Chronic maternal nicotine exposure alters neuronal systems in the arcuate nucleus that regulate feeding behavior in the newborn rhesus macaque. J. Clin. Endocrinol. Metab. 86, 5420-5426. doi: 10.1210/jcem.86.11.8033

Gual, P., Le, M.-B. Y., and Tanti, J. F. (2005). Positive and negative regulation of insulin signaling through IRS-1 phosphorylation. Biochimie 87, 99-109. doi: 10.1016/j.biochi.2004.10.019

Henkin, L., Zaccaro, D., Haffner, S., Karter, A., Rewers, M., Sholinsky, P., et al. (1999). Cigarette smoking, environmental tobacco smoke exposure and insulin 
sensitivity: the Insulin resistance atherosclerosis study. Ann. Epidemiol. 9, 290-296. doi: 10.1016/S1047-2797(99)00003-4

Holbrook, B. D. (2016). The effects of nicotine on human fetal development. Birth. Defects. Res. C. Embryo. Today. 108, 181-192. doi: 10.1002/bdrc.21128

Holloway, A. C., Lim, G. E., Petrik, J. J., Foster, W. G., Morrison, K. M., and Gerstein, H. C. (2005). Fetal and neonatal exposure to nicotine in Wistar rats results in increased beta cell apoptosis at birth and postnatal endocrine and metabolic changes associated with type 2 diabetes. Diabetologia 48, 2661-2666. doi: 10.1007/s00125-005-0022-5

Huang, T., Yang, M., Zeng, Y., Huang, X., Wang, N., Chen, Y., et al. (2021). Maternal high fat diet consumption exaggerates metabolic disorders in mice with cigarette-smoking induced intrauterine undernutrition. Front. Nutr. 8:638576. doi: 10.3389/fnut.2021.638576

Jaddoe, V. W., De Jonge, L. L., Van Dam, R. M., Willett, W. C., Harris, H., Stampfer, M. J., et al. (2014). Fetal exposure to parental smoking and the risk of type 2 diabetes in adult women. Diabetes Care 37, 2966-2973. doi: 10.2337/dc13-1679

Jamshed, L., Perono, G. A., Jamshed, S., and Holloway, A. C. (2020). Early life exposure to nicotine: postnatal metabolic, neurobehavioral and respiratory outcomes and the development of childhood cancers. Toxicol. Sci. 178, 3-15. doi: 10.1093/toxsci/kfaa127

Ko, T. J., Tsai, L. Y., Chu, L. C., Yeh, S. J., Leung, C., Chen, C. Y., et al. (2014). Parental smoking during pregnancy and its association with low birth weight, small for gestational age, and preterm birth offspring: a birth cohort study. Pediatr. Neonatol. 55, 20-27. doi: 10.1016/j.pedneo.2013.05.005

La Merrill, M. A., Cirillo, P. M., Krigbaum, N. Y., and Cohn, B. A. (2015). The impact of prenatal parental tobacco smoking on risk of diabetes mellitus in middle-aged women. J. Dev. Orig. Health. Dis. 6, 242-249. doi: $10.1017 /$ S2040174415000045

Layman, D. K., Shiue, H., Sather, C., Erickson, D. J., and Baum, J. (2003). Increased dietary protein modifies glucose and insulin homeostasis in adult women during weight loss. J. Nutr. 133, 405-410. doi: 10.1093/jn/133.2.405

Li, G., Chan, Y. L., Sukjamnong, S., Anwer, A. G., Vindin, H., Padula, M., et al. (2019). A mitochondrial specific antioxidant reverses metabolic dysfunction and fatty liver induced by maternal cigarette smoke in mice. Nutrients 11:1669. doi: $10.3390 /$ nu11071669

Lisboa, P. C., De Oliveira, E., and De Moura, E. G. (2012). Obesity and endocrine dysfunction programmed by maternal smoking in pregnancy and lactation. Front Physiol 3:437. doi: 10.3389/fphys.2012.00437

Mendez, M. A., Maties, T., Carlos, F., Nuria, R. F., and Jordi, S. (2008). Maternal smoking very early in pregnancy is related to child overweight at age 5-7 y. Am J. Clin. Nutr. 87, 1906-1913. doi: 10.1093/ajcn/87.6.1906

Montgomery, S. M., and Ekbom, A. (2002). Smoking during pregnancy and diabetes mellitus in a British longitudinal birth cohort. BMJ 324:26. doi: 10.1136/bmj.324.7328.26

Morton, G., J., Cummings, D., E., Baskin, D., G., and Barsh (2006). Central nervous system control of food intake and body weight. Nature 443, 289-295. doi: $10.1038 /$ nature 05026

Nguyen, L. T., Stangenberg, S., Chen, H., Al-Odat, I., Chan, Y. L., Gosnell, M. E., et al. (2015). L-Carnitine reverses maternal cigarette smoke exposure-induced renal oxidative stress and mitochondrial dysfunction in mouse offspring. Am. J. Physiol. Renal. Physiol. 308, F689-F696. doi: 10.1152/ajprenal.00417.2014

Oelkrug, R., Polymeropoulos, E. T., and Jastroch, M. (2015). Brown adipose tissue: physiological function and evolutionary significance. J. Comp. Physiol. B. 185, 587-606. doi: 10.1007/s00360-015-0907-7

Parker, B., Noakes, M., Luscombe, N., and Clifton, P. (2002). Effect of a high-protein, high-monounsaturated fat weight loss diet on glycemic control and lipid levels in type 2 diabetes. Diabetes Care 25, 425-430. doi: $10.2337 /$ diacare.25.3.425

Peixoto, T. C., Moura, E. G., Soares, P. N., Bertasso, I. M., Pietrobon, C. B., Caramez, F., et al. (2020). Nicotine exposure during breastfeeding reduces sympathetic activity in brown adipose tissue and increases in white adipose tissue in adult rats: sex-related differences. Food. Chem. Toxicol. 140:111328. doi: $10.1016 /$ j.fct.2020.111328

Primo, C. C., Ruela, P. B., Brotto, L. D., Garcia, T. R., and Lima Ede, F. (2013). Effects of maternal nicotine on breastfeeding infants. Rev. Paul. Pediatr. 31, 392-397. doi: 10.1590/S0103-05822013000300018
Saad, S., Al-Odat, I., Chan, Y. L., Mcgrath, K. C., Pollock, C. A., Oliver, B. G., et al. (2018). Maternal L-carnitine supplementation improves glucose and lipid profiles in female offspring of dams exposed to cigarette smoke. Clin. Exp. Pharmacol. Physiol. 45, 694-703. doi: 10.1111/1440-1681.12921

Saeedi, P., Petersohn, I., Salpea, P., Malanda, B., Karuranga, S., Unwin, N., et al. (2019). Global and regional diabetes prevalence estimates for 2019 and projections for 2030 and 2045: Results from the International Diabetes Federation Diabetes Atlas, 9(th) edition. Diabetes. Res. Clin. Pract. 157:107843. doi: 10.1016/j.diabres.2019.107843

Skov, A. R., Toubro, S., Rønn, B., Holm, L., and Astrup, A. (1999). Randomized trial on protein vs carbohydrate in ad libitum fat reduced diet for the treatment of obesity. Int. J. Obes. Relat. Metab. Disord. 23, 528-536. doi: $10.1038 /$ sj.ijo.0800867

Sullivan, E. L., Nousen, E. K., and Chamlou, K. A. (2014). Maternal high fat diet consumption during the perinatal period programs offspring behavior. Physiol. Behav. 123, 236-242. doi: 10.1016/j.physbeh.2012. 07.014

Sun, X., and Zemel, M. B. (2007). Leucine and calcium regulate fat metabolism and energy partitioning in murine adipocytes and muscle cells. Lipids 42, 297-305. doi: 10.1007/s11745-007-3029-5

Talhout, R., Schulz, T., Florek, E., Van Benthem, J., Wester, P., and Opperhuizen, A. (2011). Hazardous compounds in tobacco smoke. Int. J. Environ. Res. Public. Health. 8, 613-628. doi: 10.3390/ijerph8020613

Vlahos, R., Bozinovski, S., Jones, J. E., Powell, J., Gras, J., Lilja, A., et al. (2006). Differential protease, innate immunity and NF kappa B induction profiles during lung inflammation induced by sub-chronic cigarette smoke exposure in mice. Am. J. Physiol. Lung. Cell. Mol. Physiol. 290, L931-L945. doi: 10.1152/ajplung.00201.2005

Vuguin, P., Raab, E., Liu, B., Barzilai, N., and Simmons, R. (2004). Hepatic insulin resistance precedes the development of diabetes in a model of intrauterine growth retardation. Diabetes 53, 2617-2622. doi: 10.2337/diabetes.53. 10.2617

Wagijo, M. A., Sheikh, A., Duijts, L., and Been, J. V. (2017). Reducing tobacco smoking and smoke exposure to prevent preterm birth and its complications. Paediatr. Respir. Rev. 22, 3-10. doi: 10.1016/j.prrv.2015.09.002

Westerterp-Plantenga, M. S., Nieuwenhuizen, A., Tom,é, D., Soenen, S., and Westerterp, K. R. (2014). Dietary protein, weight loss, and weight maintenance. Curr. Opin. Clin. Nutr. Metab. Care. 17, 75-79. doi: 10.1146/annurev-nutr-080508-141056

World Health Organization (2019). WHO Global Report on Trends in Prevalence of Tobacco Use 2000-2025, 3rd Edn. Available online at: https://www.who.int/ news/item/19-12-2019-who-launches-new-report-on-global-tobacco-usetrends

Yang, J., Chi, Y., Burkhardt, B. R., Guan, Y., and Wolf, B. A. (2010). Leucine metabolism in regulation of insulin secretion from pancreatic beta cells. Nutr. Rev. 68, 270-279. doi: 10.1111/j.1753-4887.2010. 00282.x

Zhang, Y., Guo, K., Leblanc, R. E., Loh, D., Schwartz, G. J., and Yu, Y. H. (2007). Increasing dietary leucine intake reduces diet-induced obesity and improves glucose and cholesterol metabolism in mice via multimechanisms. Diabetes 56, 1647-1654. doi: 10.2337/db07-0123

Zheng, Y., Ley, S. H., and Hu, F. B. (2018). Global aetiology and epidemiology of type 2 diabetes mellitus and its complications. Nat. Rev. Endocrinol. 14, 88-89. doi: $10.1038 /$ nrendo.2017.151

Conflict of Interest: The authors declare that the research was conducted in the absence of any commercial or financial relationships that could be construed as a potential conflict of interest.

Copyright $\odot 2021$ Zeng, Huang, Wang, Xu, Sun, Huang, Chen, Oliver, Yi and Chen. This is an open-access article distributed under the terms of the Creative Commons Attribution License (CC BY). The use, distribution or reproduction in other forums is permitted, provided the original author(s) and the copyright owner(s) are credited and that the original publication in this journal is cited, in accordance with accepted academic practice. No use, distribution or reproduction is permitted which does not comply with these terms. 